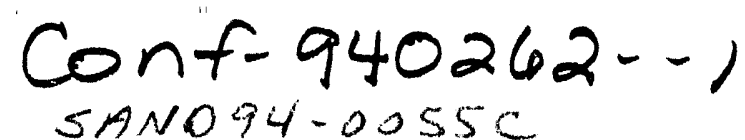

\title{
TEMPERATURE DEPENDENCE OF THE TWO-PHOTON ABSORPTION' SPECTRUM OF POLY(DI-N-HEXYLSILANE)
}

\author{
R. G. KEPLER \\ Sandia National Laboratories, Albuquerque, NM 87185 \\ and \\ Z. G. SOOS \\ Department of Chemistry, Princeton University, Princeton, NJ 08544
}

\begin{abstract}
We have measured the temperature dependence of the intensity of the lowest energy two-photon absorption line in poly(di-nhexylsilane) and find that it does not change between ambient temperature and $14 \mathrm{~K}$. The line width decreases by about a factor of three.
\end{abstract}

\section{INTRODUCTION}

The recent demonstration that light emitting diodes could be made by placing a spin-cast conjugated polymer film which fluoresces between two electrodes ${ }^{1}$ has emphasized the importance of activity desigried to understand the nature of the excited states of conjugated polymers. We have been studying excited states in polysilanes, $\sigma$-conjugated, silicon-backbone polymers, ${ }^{2}$ and using this class of polymers as models for conjugated polymers in which the transferintegral alternation and band gap are large, and which fluoresce with a high quantum efficiency. We have shown that the electronic states of these polymers, as well as those of the more familiar $\pi$-conjugated polymers, can be described by the Hückel model if coulomb interactions are included using the Pariser-Parr-Pople approximation. ${ }^{3-5}$

Recently we reported ${ }^{6}$ that the intensity of the two-photon transition at $11 \mathrm{~K}$ was an order of magnitude greater than that at ambient temperature in poly(di-n-hexylsilane), a crystalline polymer. That observation led Mukamel ${ }^{7}$ to point out that if in fact that was true it would provide strong support for the exciton model of Leegwater and Mukamel. ${ }^{8}$ We therefore undertook a careful examination of the temperature dependence of the two-photon transition in thin films of poly(di-n-hexylsilane), PDHS.

In this paper we report that between ambient temperature and $12 \mathrm{~K}$ the fluorescence quantum efficiency of PDHS increases more than a factor of two, 


\section{R. G. KEPLER AND Z. G. SOOS}

the fluorescence line width narrows by about a factor of two, and the twophoton absorption line width decreases by about a factor of three, but the area under the two-photon absorption band does not change, within the accuracy of our experiments. These results, therefore, do not provide support for the Leegwater and Mukamel theory as the earlier report suggested they would. The different conclusion reported in the earlier work resulted from monitoring the intensity of the fluorescence induced by photons at the peak of the twophoton absorption band by measuring the intensity at the peak of the fluorescence band. The change in the area under the two spectra was not taken into account and no correction was made for the change in fluorescence quantum efficiency with temperature.

\section{EXPERIMENT}

Two thin film samples were used for these studies, one about $1 \mu \mathrm{m}$ was solution cast from a toluene solution and the other about $0.1 \mu \mathrm{m}$ was spin cast from a toluene solution. The substrate for both samples was a $0.1 \mathrm{~mm}$ s?pphire flat. At low temperatures, thick PDHS samples crack and become detached from the substrate. To prevent pieces from falling off, both samples were covered by a second sapphire flat. A Nd:YAG-laser-pumped dye-laser was used to excite the samples and the intensity of the sample fluorescence was measured with a monochromator-1P28 photomultiplier combination. The temperature dependence of the absorption coefficient was determined by measuring the wavelength dependence of the amount of light from a xenon arc-monochromator combination transmitted by the $0.1 \mu \mathrm{m}$ sample at ambient temperature and $12 \mathrm{~K}$.

In order to determine the temperature dependence of the quantum efficiency for fluorescence, the samples were excited at $355 \mathrm{~nm}$ for the thick sample and at $297 \mathrm{~nm}$ for the thin sample, and the intensity of the fluorescent light was measured as a function of wavelength and temperature. The quantum efficiency for fluorescence was assumed to be proportional to the number of fluorescent photons detected divided by the number of exciting photons absorbed. All of the photons incident on the thick sample were absorbed at all temperatures and the number of photons absorbed in the thin sample was calculated from the measured temperature dependent absorption spectrum.

The two-photon absorption spectrum was measured by first measuring the fluorescence intensity a some arbitrary wavelength as a function of the exciting light wavelength. These data were used to determine the peak of the two-photon absorption band at the temperature of the sample. The fluorescence spectrum was then measured with the exciting light wavelength set at the maximum of the two-photon absorption band. Finally, the intensity of fluorescence at the peak of the fluorescence spectrum was measured as a 
function of the exciting wavelength to determine the shape and intensity of the two-photon absorption band.

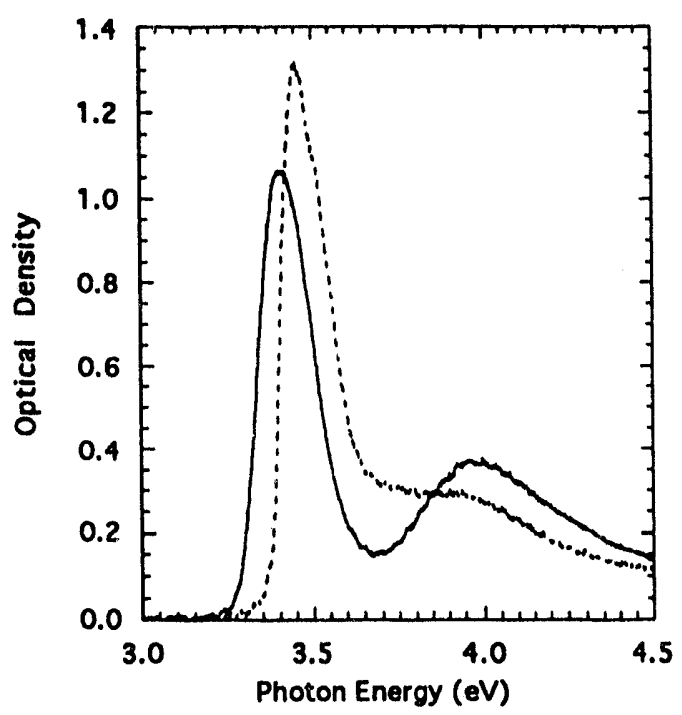

Fig. 1. Optical density of a $100 \mathrm{~nm}$ film of PDHS at $295 \mathrm{~K}$ (solid line) and $14 \mathrm{~K}$ (dashed line)
A single dye was used in the dye laser for these measurements so that we were unable to determine the full two-photon absorption spectrum. The full spectrum was approximated by using a least squares fit of a Gaussian line to the limited data in the vicinity of the peak of the absorption band. We subsequently used the area under the Gaussian curve as a measure of the two-photon absorption intensity. We did not change dyes in the laser because it would be very time consuming and would probably have introduced larger errors than the approximation procedure we used because of the many adjustments required when dyes are changed.

\section{$\underline{\text { RESULTS }}$}

The one-photon absorption spectrum of PDHS obtained at ambient temperature and at $12 \mathrm{~K}$ on the $0.1 \mu \mathrm{m}$ sample are shown in Fig. 1 . The width of the main, lowest energy absorption band does not change appreciably. An unexplained blue shift in the energy of the transition at low temperatures, a shift that has been noted a number of times ${ }^{9}$, is readily evident. The second peak which is usually ascribed to a remnant of a high temperature phase is seen to decrease.

The fluorescence spectrum of the thin sample at $295 \mathrm{~K}, 154 \mathrm{~K}$ and $14 \mathrm{~K}$ are shown in Fig. 2. Similar data were obtained on the thick film. In both experiments the fluorescent light was observed from the side of the sample opposite to that excited. For the thin sample the amount of light emitted per absorbed photon at $14 \mathrm{~K}$ was 2.25 times the amount emitted at $295 \mathrm{~K}$. For the thick sample the same ratio was 3.0. In both samples the fluorescence line narrowed at $14 \mathrm{~K}$ relative to the width at ambient temperature, by almost a factor two in the thin sample and by $20 \%$ in the thick sample. The blue shift in 


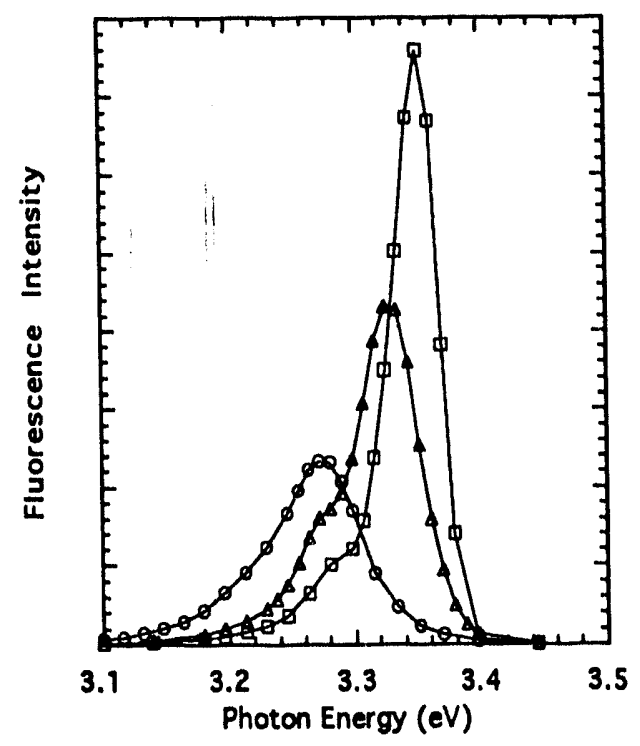

Fig. 2. Fluorescence spectrum of a $100 \mathrm{~nm}$ film of PDHS at $295 \mathrm{~K}$ (circles), $154 \mathrm{~K}$ (triangles) and $14 \mathrm{~K}$ (squares). The solid lines are quides for the eye. the fluorescence spectrum was comparable to that observed in the absorption spectrum.

The two-photon absorption spectrum obtained on the thin film is shown in Fig. 3. Two dyes were used to cover the complete absorption line at this temperature. The blueshift in the peak of the line was $0.05 \mathrm{eV}$ compared to 0.07 to $0.10 \mathrm{eV}$ for the absorption and fluorescence lines, and the line narrowed by about a factor of 3 between $295 \mathrm{~K}$ and $14 \mathrm{~K}$. Essentially the same blue-shift and line narrowing were observed in the thick sample. These results are nearly identical to those reported by us previously. ${ }^{6}$

After correcting for the change with temperature of the fluorescence quantum efficiency, the experimental results on the thick film indicated that the twophoton transition became stronger by a factor of two at $14 \mathrm{~K}$ relative to $295 \mathrm{~K}$. The thin film experimental results indicated that the transition strength at $14 \mathrm{~K}$ was $85 \%$ of that at $295 \mathrm{~K}$. Therefore, we conclude that within the accuracy of our experiment there is no change between ambient temperature and $14 \mathrm{~K}$.

\section{DISCUSSION}

It is generally accepted that the absorption spectrum of the polysilanes is inhomogeneously broadened. The long polymer chain is believed to be divided into chain segments of random length by undefined defects and the energy of the optical transition depends on the segment length. ${ }^{10}$ Therefore, the fact that the width of the lowest energy transition is essentially independent of temperature is not surprising. The blue shift in the energy of the transition is surprising and it has not been explained.

A red shift is clearly expected if increased ordering results in longer segments at low temperature. Smaller unit-cell volumes and stronger Van der Waals interactions also lead to a red shift. Blue shifts can result from a 


\section{TEMPERATURE DEPENDENCE OF TWO-PHOTON ABSORPTION}

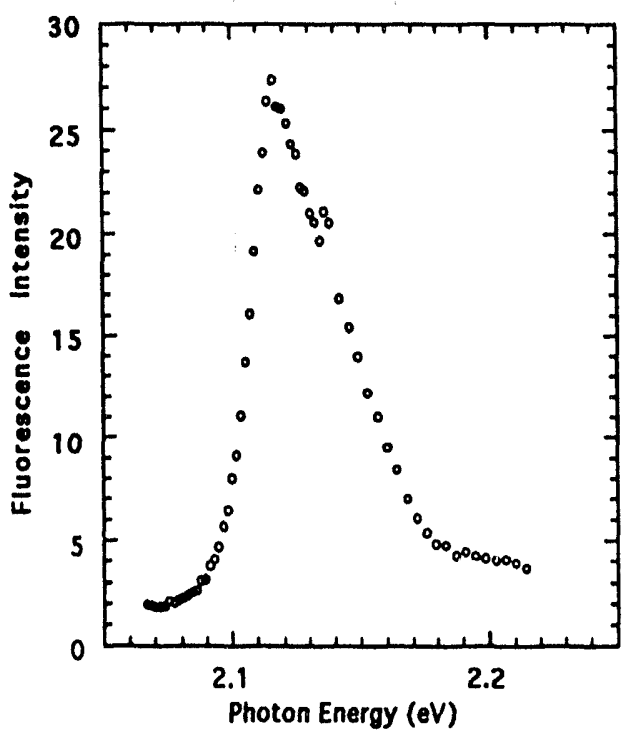

Fig. 3. The two-photon absorption spectrum of a $100 \mathrm{~nm}$ thick film of PDHS at $14 \mathrm{~K}$. decrease in the Si-Si-Si bond angle as the contribution of thermal fluctuations to steric hindrance decrease at low temperature. Harrah and Zeigler have shown that an increase in steric hindrance leads to a lower transition energy. ${ }^{11}$ They can also result from third-neighbor $\mathrm{Si}$ interations. ${ }^{12}$

The fact that the polysilanes fluoresce at an energy lower than the absorption band is usually attributed to energy transfer from higher energy segments to lower energy segments prior to photon emission. Some have suggested that the excitons become trapped in long, low energy

segments, but we recently showed that they remain mobile throughout their lifetime, even at low temperatures. ${ }^{13,14}$ Therefore it is surprising that the fluorescence line width does not decrease more at low temperatures.

Perhaps the feature of these spectra that is the most difficult to explain is the factor of three change in the width of the two-photon absorption band when a similar change is not seen in the absorption band. Our model of the two-photon transition ${ }^{3}$ suggests that, to a first approximation, the two-photon absorption band should parallel the behavior of the one-photon absorption band. One major difference is that the strength of one photon transitions increases linearly with segment length while the strength of two photon transitions increases quadratically with segment length, up to some conjugation length on the order of 20-30 $\mathrm{Si}$ atoms. If the length of the ordered segments increase with decreasing temperature, a narrowing of the twophoton line relative to the one-photon line would occur, but then the strength of the two photon line should increase and the energy of the transitions should decrease. The fact that the energy of the transitions increase rather than decrease could be rationalized by assuming that the steric hindrance, or whatever effect causes the blue shift, overwhelms the red shift but that effect would not explain the lack of an increase in the strength of the two-photon transition. 
R. G. KEPLER AND Z. G. SOOS

\title{
ACKNOWLEDGMENTS
}

The technical assistance of P. M. Beeson is gratefully acknowledged. The work at Sandia National Laboratories was supported by the U. S. Department of Energy under Contract No. DE-AC04-76DP00789 and the work at Princeton University was partially supported by NSF Grant No. NSF-DMR-8921072.

\section{REFERENCES}

1. J. H. Burroughes, D. D. C. Bradley, A. R. Brown, R. N. Marks, K. Mackay, R. H. Friend, P. L. Burns and A. B. Holmes, Nature 347, 539 (1990).

2. R. G. Kepler and Z. G. Soos, in Relaxation in Polymers, edited by T. Kobayashi, (World Scientific, Singapore, 1993)

3. Z. G. Soos and G. W. Hayden, Chem. Phys. 143, 199 (1990).

4. Z. G. Soos and S. Ramasesha, Phys. Rev. B 29, 5410 (1984).

5. Z. G. Soos, S. Ramasesha and D. S. Galvao, Phys. Rev Lett. 71, 1609 (1993).

6. Z. G. Soos and R. G. Kepler, Phys. Rev. B 43 3 11908 (1991).

7. S. Mukamel, personal communication

8. J. A. Leegwater and S. Mukamel, Phys. Rev. A 46,452 (1992).

9. R. D. Miller and J. Michl, Chem. Rev. 89 , 1359 (1989).

10. K. S. Schweizer, in Silicon-Based Polymer Science: A Comprehensive Resource, edited by J. M. Zeigler and F. W. G. Fearon, Advances in Chemistry Series 224 (American Chemical Society, Washington DC, 1990) p. 379.

11. L. A. Harrah and J. M. Zeigler, Macromolecules 20, 601 (1987).

12. V. Balaji and J. Michl, Polyhedron 101265 (1991).

13. R. G. Kepler and Z. G. Soos, Phys. Rev. B 47, 9253 (1993).

14. R. G. Kepler and Z. G. Soos, (to be published).

\section{DISCLAIMER}

\begin{abstract}
This report was prepared as an account of work sponsored by an agency of the United States Government. Neither the United States Government nor any agency thereof, nor any of their employees, makes any warranty, express or implied, or assumes any legal liability or responsibility for the accuracy, completeness, or usefulness of any information, apparatus, product, or process disclosed, or represents that its use would not infringe privately owned rights. Reference herein to any specific commercial product, process, or service by trade name, trademark, manufacturer, or otherwise does not necessarily constitute or imply its endorsement, recommendation, or favoring by the United States Government or any agency thereof. The views and opinions of authors expressed herein do not necessarily state or reflect those of the United States Government or any agency thereof.
\end{abstract}



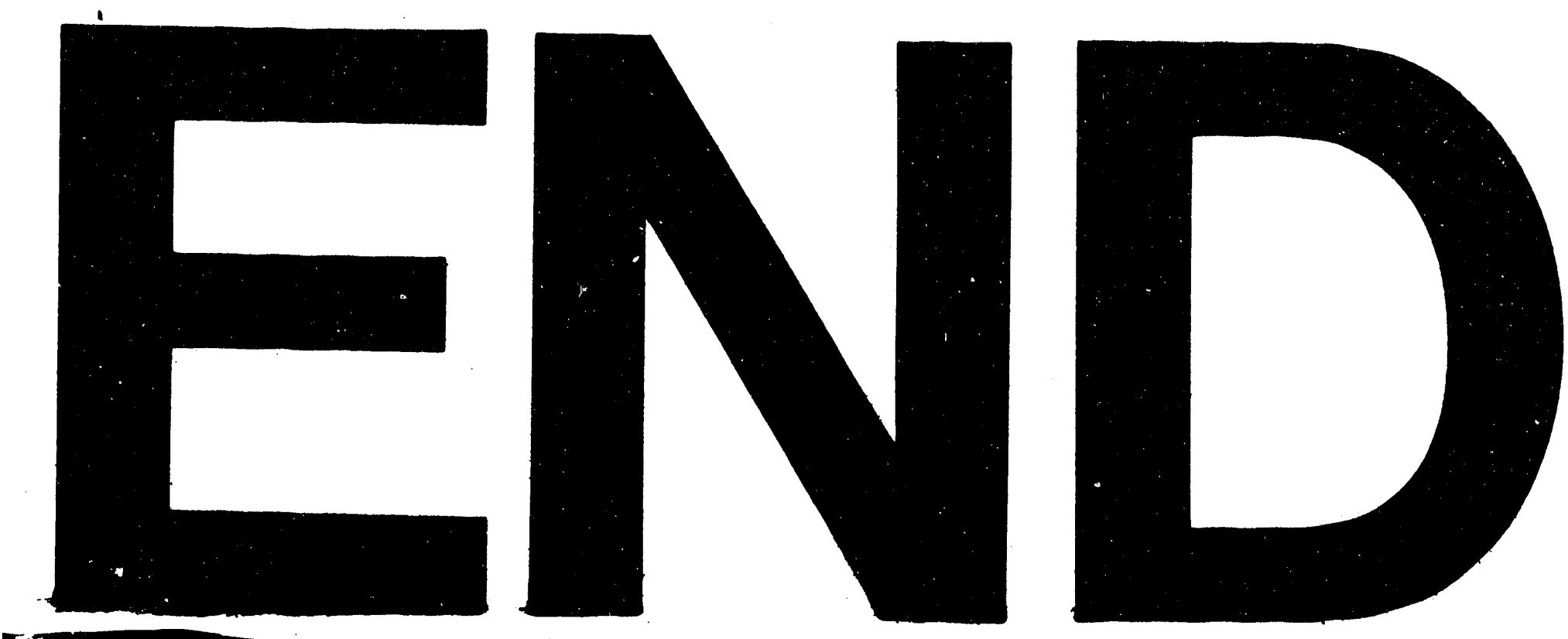

5

$x$
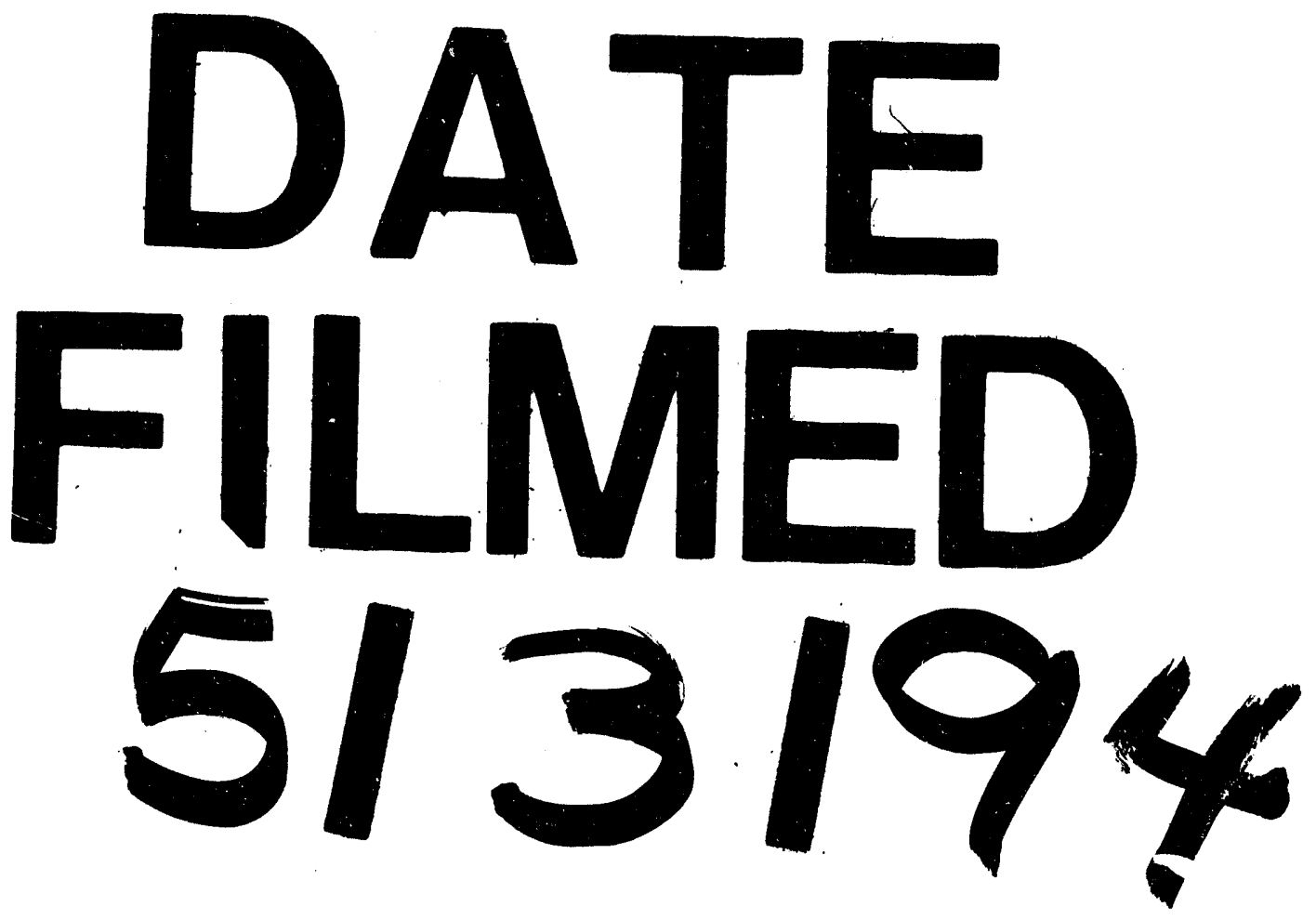


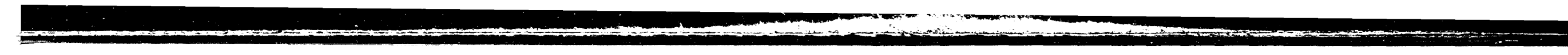

